\title{
Sensorimotor Rhythm Neurofeedback Enhances Golf Putting Performance
}

\author{
Ming-Yang Cheng, ${ }^{1}$ Chung-Ju Huang, ${ }^{2}$ Yu-Kai Chang, ${ }^{3}$ \\ Dirk Koester, ${ }^{1}$ Thomas Schack, ${ }^{1}$ and Tsung-Min Hung ${ }^{4}$ \\ ${ }^{1}$ Bielefeld University; ${ }^{2}$ University of Taipei; ${ }^{3}$ National Taiwan Sport University; \\ ${ }^{4}$ National Taiwan Normal University
}

\begin{abstract}
Sensorimotor rhythm (SMR) activity has been related to automaticity during skilled action execution. However, few studies have bridged the causal link between SMR activity and sports performance. This study investigated the effect of SMR neurofeedback training (SMR NFT) on golf putting performance. We hypothesized that preelite golfers would exhibit enhanced putting performance after SMR NFT. Sixteen preelite golfers were recruited and randomly assigned into either an SMR or a control group. Participants were asked to perform putting while electroencephalogram (EEG) was recorded, both before and after intervention. Our results showed that the SMR group performed more accurately when putting and exhibited greater SMR power than the control group after 8 intervention sessions. This study concludes that SMR NFT is effective for increasing SMR during action preparation and for enhancing golf putting performance. Moreover, greater SMR activity might be an EEG signature of improved attention processing, which induces superior putting performance.
\end{abstract}

Keywords: precision sports, attention, EEG, sensorimotor rhythm, automaticity

The quality of mental regulation can differentiate superior from inferior performance in precision sports activities such as golf putting. In golf, the putt is considered one of the most important parts of the game, representing on average $43 \%$ of all shots taken during a single round (Pelz \& Frank, 2000). From a technical perspective, putting is the simplest skill used in golf. However, mentally, putting is the most stressful and demanding activity in the game (Nicholls, 2007). The mental challenge of putting is reflected by previous psychophysiological studies showing complex brain processes during putting performance (Babiloni et al., 2008). Hence, the maintenance

\footnotetext{
Ming-Yang Cheng is with Center of Excellence "Cognitive Interaction Technology" (CITEC), Bielefeld University, Bielefeld, Germany. Chung-Ju Huang is with the Graduate Institute of Sport Pedagogy, University of Taipei, Taipei City, Taiwan, Republic of China. Yu-Kai Chang is with the Graduate Institute of Athletics and Coaching Science, National Taiwan Sport University, Taoyuan County, Taiwan, Republic of China. Dirk Koester is with the Center of Excellence "Cognitive Interaction Technology" (CITEC), Bielefeld University, Bielefeld, Germany. Thomas Schack is with the Center of Excellence "Cognitive Interaction Technology" (CITEC), Bielefeld University, Bielefeld, Germany. Tsung-Min Hung is with the Department of Physical Education, National Taiwan Normal University, Taipei City, Taiwan, Republic of China. Address author correspondence to Tsung-Min Hung at ernesthungkimo@yahoo.com.tw.
}

of a mental state conducive to skilled execution is critical for ideal precision sports performance.

Superior performance in precision sports can be characterized as an automatic process as opposed to a controlled process, which is typically observed in less skilled performers (Fitts \& Posner, 1967). An automatic process is by nature reflexive, whereas a controlled process is an intentionally initiated sequence of cognitive activity (Schneider \& Shiffrin, 1977). Achieving automatic process in action execution is the primary goal of mastery (Logan, Hockley, \& Lewandowsky, 1991). Differences between these two levels of cognitive processing are reflected at the neurophysiological level: participants who were in the automatic stage exhibited weaker activity of the bilateral cerebellum, presupplementary motor area, premotor cortex, parietal cortex, and prefrontal cortex compared with novices (Wu, Chan, \& Hallett, 2008). In addition, the somatosensory cortex has been related to conscious perception of somatosensory stimuli (Nierhaus et al., 2015), such that lower activity in the somatosensory cortex might be a signature of reduced conscious involvement in movement execution, as is frequently observed in highly skilled performers.

Although previous studies of the brain function underlying superior golf putting performance have provided insights into adaptive mental states and their cortical processes, few studies have examined the cortical processes that are more directly associated with somatosensory activity. For example, Babiloni 
et al. (2008) demonstrated that successful putting was preceded by higher high-frequency alpha (10-12 Hz) event-related desynchronization over the frontal midline and the right primary sensorimotor area compared with unsuccessful putting performance. Similarly, studies found that reduced (Kao, Huang, \& Hung, 2013) and stable (Chuang, Huang, \& Hung, 2013) frontal midline theta power was the precursor of superior performance in precision sports. Since high-frequency alpha power in these cortical areas reflect only task-related attention (Klimesch, Doppelmayr, Pachinger, \& Ripper, 1997) whereas frontal midline theta power indicates top-down sustained attention (Sauseng, Hoppe, Klimesch, Gerloff, \& Hummel, 2007), these findings support the importance of specialized task-related attention on superior motor performance. However, the information encoded during automatic somatosensory processing during skilled precision sport performance remains unexamined as yet.

Sensorimotor rhythm (SMR), the 12- to $15-\mathrm{Hz}$ oscillation of the sensorimotor cortex, has shown promising as a link between adaptive mental states (e.g., automatic process-related attention) and skilled visuomotor performance. Sensorimotor rhythm is considered an indicator of cortical activation, which is inversely related to somatosensory processing (Mann, Sterman, \& Kaiser, 1996). A recent study showed that skilled dart-throwing players demonstrated higher SMR power before dart release than novices in a dart-throwing task (Cheng et al., 2015). This result suggests that lower cognitive involvement in processing somatosensory information as reflected by higher SMR power is characteristic of skilled performance. Furthermore, several lines of studies pertaining to SMR power tuning for enhancing adaptive cortical processing in motor performance have shown promising results. Augmented SMR power resulting from neurofeedback training (NFT) has been identified as a relaxed focus state without somatosensory intervention (Gruzelier, Foks, Steffert, Chen, \& Ros, 2014). Similarly, a reduced trait anxiety score and task-processing time during microsurgery were observed after augmented SMR NFT (Ros et al., 2009). Moreover, a facilitative sense of control, confidence, and feeling at-one with a role was demonstrated after augmented SMR NFT before acting performance (Gruzelier, Inoue, Smart, Steed, \& Steffert, 2010). Thus, increased SMR activity implies the maintenance of a relaxed, focused state by reducing motor perception (e.g., somatosensory processing) by the sensorimotor cortex (Vernon et al., 2003). This interpretation is similar to the mental characteristics of peak performance in skilled athletes (Krane \& Williams, 2006) and is in agreement with the concept of automaticity proposed by Fitts and Posner (1967). Hence, SMR power not only might be a sensitive indicator of the activity of sensorimotor cortex (Mann et al., 1996) but also shows potential for a performance-enhancing intervention.

Although there is no direct evidence to support the effectiveness of SMR NFT on performance enhancement in precision sport, two lines of research lend support to its potential use in sports. First, previous studies have demonstrated the effectiveness of NFT on performance enhancement in precision sports. For example, Landers et al. (1991) demonstrated that "correct" NFT (i.e., augmented slow cortical potential at the left temporal lobe) led to superior performance, whereas "incorrect" NFT (i.e., augmented slow cortical potential at the right temporal lobe) impaired performance in skilled archers. Similarly, Kao, Huang, and Hung (2014) reported that NFT targeting to reduce the frontal midline theta resulted in improved performance in skilled golfers. These findings support the feasibility of tuning EEG to improve behavioral outcome in precision sports. The second line of evidence is the finding that SMR NFT has a beneficial effect on attention-related performance in various attentional tasks. For example, an increased P300b amplitude at frontal, central, and parietal sites during the auditory oddball task and reduced commission errors, and a reduction in reaction time variability during the Test of Variables of Attention (TOVA) was observed after augmented SMR NFT (Egner, Zech, \& Gruzelier, 2004).

These findings suggest that augmenting SMR power might improve attention-related processes by improving impulse control and the ability to integrate relevant environmental stimuli. Similarly, Ros et al. (2009) reported that a shorter operation time and reduced trait anxiety score were observed in surgeons following augmented SMR NFT, suggesting that augmented SMR enhanced the learning of a complex medical specialty by developing sustained attention and a relaxed attentional focus as well as increasing working memory (Vernon et al., 2003). Furthermore, Doppelmayr and Weber (Doppelmayr \& Weber, 2011) revealed that augmented SMR NFT not only resulted in a significant SMR amplitude increase accompanied by a significant increase in reward threshold, but also facilitated the performance of spatialrotation, simple, and choice-reaction time tasks. These results indicate that visuospatial processing, semantic memory regulation, and the integration of relevant stimuli can be improved following augmented SMR NFT. Collectively, the benefits of augmented SMR NFT can be attributed to an improved regulation of somatosensory and sensorimotor pathways, which in turn leads to more efficient attention allocation (Kober et al., 2014) that results in an improved processing of task-relevant stimuli.

To the best of our knowledge, no study has directly examined the effect of SMR NFT on precision sport performance. Thus, this study investigated the effect of SMR NFT on a golf putting task. We predicted that golfers would be able to increase SMR power before putting execution following augmented SMR NFT. More importantly, we predicted that increased SMR power improves putting performance as a result of augmented SMR NFT.

\section{Methods}

\section{Participants}

Fourteen male and two female preelite and elite golfers were recruited (mean handicap $=0, S D=3.90$ ). 
Participants were matched based on performance history supplemented by the assessment of a professional coach and then randomly assigned into either an SMR neurofeedback group (SMR NFT) or a control group (seven male and one female for each group). The mean age of the SMR NFT and control group were 20.6 (1.59) and 22.3 (2.07), respectively. The years of experience in golf were 9.5 (2.67) for the SMR NFT group and 9.2 (1.83) for the control group. An independent $t$ test showed no difference in age $[t(14)=1.895$, $p=.079]$ or years of experience in golf $[t(14)=0.273, p$ $=.789$ ] between the two groups. None of the participants reported psychiatric and neurological disorders and had never been hospitalized for general brain damage.

\section{Procedures}

For the pretest and posttest, we used the same procedure to collect data. At pretest, after being informed of the general purpose of the study, all participants were asked to read and sign an informed consent form approved by our institutional review board. They were then given the opportunity to ask questions about the experiment. The participants were individually tested in a sound-proof indoor artificial golf green, where they were initially required to stand $3 \mathrm{~m}$ from a hole $10.8 \mathrm{~cm}$ in diameter to obtain an individual putting distance (Arns, Kleinnijenhuis, Fallahpour, \& Breteler, 2008). Participants performed a series of 10 putts, which were scored as successfully holed or not holed. The percentage of successful putts in a series was determined after each series. This process was repeated until each participant achieved 50\% accuracy.

After the individual putting distance was determined, participants were fitted with a Lycra electrode cap (Neuroscan, Charlotte, NC, USA). After a 10-min warm-up, participants were first asked to undergo a resting EEG recording, including eye-closed and eye-opened conditions, while assuming a normal putting stance for $1 \mathrm{~min}$ each. Then, all participants performed golf putting tasks consisting of 40 self-paced putting trials in four separate recording blocks while EEGs were recorded. The participants performed the putting task in the standing position and were allowed to take a brief rest between each putt. They were also allowed to sit briefly after each block of 10 putts. The score was calculated based on the linear distance from the edge of hole to the edge of the ball $(\mathrm{cm})$. Putting into the hole successfully was determined as score 0 . Putting trials in which the ball was deflected by contacting the edge of the hole were excluded, and participants were asked to perform extra putting trials to complete the forty trials. The experiment lasted approximately $2 \mathrm{hr}$ in total. After completing the pretest, all participants were scheduled to go through 8 sessions of neurofeedback training. Then the posttest, which was identical to the pretest, followed the neurofeedback intervention.

\section{Instrumentation}

Electroencephalography. For the pretest and posttest, EEGs were recorded at 32 electrode sites (FP1, FP2, F7,
F8, F3, F4, FZ, FT7, FT8, FC3, FC4, C3, C4, CZ, T3, T4, T5, T6, TP7, TP8, CP3, CP4, CPZ, A1, A2, P3, P4, PZ, $\mathrm{O} 1, \mathrm{O} 2, \mathrm{OZ})$ corresponding to the International 10-10 system (Chatrian, Lettich, \& Nelson, 1985). In addition, four electrodes were attached to acquire horizontal and vertical oculography (HEOL, HEOR, VEOU and VEOL). All sites were initially referenced to $A 1$ and then rereferenced to linked ears offline. A frontal midline site (FPz) served as the ground. EEG data were collected and amplified using a Neuroscan Nuamps amplifier (Neuroscan, Charlotte, NC, USA) with a band-pass filter setting of $1-100 \mathrm{~Hz}$ and a $60-\mathrm{Hz}$ notch filter. The EEG and EOG signals were sampled at $500 \mathrm{~Hz}$ and recorded online with NeuroScan 4.5 (Neuroscan, Charlotte, NC, USA) software installed on a Lenovo R400 laptop (Lenovo, Taipei City, R.O.C). Vertical and horizontal eye movement artifacts were recorded via bipolar electro-oculographic activity (EOG), in which vertical EOG was assessed by electrodes placed above and below the left eye (VEOU and VEOL), whereas horizontal EOG was assessed by electrodes located at the outer canthi (HEOL, HEOR). Impedance values for all electrode sites were maintained below $5 \mathrm{k} \Omega$. An infrared ray sensor was set to detect the swing for each putt. Once the back swing movement was detected, an event mark was sent to the EEG data, which served as the time point for analyzing the EEG activity before putting. Twelve to fifteen hertz of $\mathrm{Cz}$ was extracted as the SMR (Babiloni et al., 2008).

Neurofeedback. Neurofeedback training was completed with a NeuroTek Peak Achievement Trainer (NeuroTek, Goshen, KY). The EEG data from the assessment were band-pass filtered using the BioReview software (NeuroTek, Goshen, KY). The active scalp electrode was placed at $\mathrm{Cz}$ for SMR training, with the reference placed on both mastoids. Signal was acquired at $256 \mathrm{~Hz}$ and then A/D converted and band filtered to extract the SMR $(12-15 \mathrm{~Hz})$. The amplitude of the SMR was transformed online into graphical feedback representations including the low-frequency audio-feedback tone by acoustic bass (No. 33) in the BioReview software.

\section{Neurofeedback Training Procedure}

Participants underwent an eight-session training program lasting 5 weeks. Each session was composed of neurofeedback training lasting from 30 to $45 \mathrm{~min}$. On average, a total of 12 training trials were performed in a single session. Each training trial comprised $30 \mathrm{~s}$. The total duration of a single session was approximately $30 \mathrm{~min}$. The SMR NFT group aimed to increase absolute SMR amplitude over the designated threshold, which was individually determined by averaging $1.5 \mathrm{~s}$ of each participant's successful putting trials during the pretest. To enhance the participants' efficacy during NFT, a progressive adjustment of the training threshold difficulty was employed. The standard for adjusting the training threshold was based on the individualized standard deviation which derived from the SMR power of the final three 0.5-s time windows before putting during the pretest. When 
participants' SMR power was higher than the threshold, the acoustic bass sound was displayed. Participants were instructed to perform based on their own putting routine while receiving the auditory feedback. The successful training ratio, defined as the time spent above threshold divided by the total time of a single training trial (30 s), was reported to participants following every training trial.

In the control group, the training protocol was similar to that used by Egner, Strawson, and Gruzelier (2002) to establish a mock feedback condition. This protocol was designed to prevent study participants from learning to regulate SMR by using the randomly prerecorded feedback tone during the training trials from SMR NFT group. The total length of this prerecorded mock feedback tone was 4 min that were derived from a randomly chosen participant in the SMR NFT group during the Session 1 training. Researchers played the mock feedback tone from a random starting point to guarantee a randomized feedback tone was received by participants in the control group. On average, a total of seven training trials were performed in a single session and the total duration of a single session was approximately $30 \mathrm{~min}$.

To evaluate the neurofeedback learning effect, the mean successful training ratio of each session was recorded and computed for subsequent analysis. To reduce the number of sessions necessary for statistical evaluation of the learning efficiency between the two groups, we combined two consecutive sessions into one section [e.g., Section $1=($ Session $1+$ Session 2$) / 2$ ].

\section{Data Reduction}

The EEG data reduction was conducted offline using the Scan 4.5 software (Neuroscan, Charlotte, NC, USA). EEG data were sampled $1.5 \mathrm{~s}$ before putting execution and were triggered by the event-related marker from infrared ray sensors. Trial preparation periods of less than $1.5 \mathrm{~s}$ were excluded to establish the common structure of artifact-free data across trials and participants. EOG correction (Semlitsch, Anderer, Schuster, \& Presslich, 1986) was carried out on continuous EEG data to eliminate blink artifacts. EEG segments with amplitudes exceeding \pm 100 $\mu \mathrm{V}$ from baseline were excluded from subsequent analysis. After artifact-free EEG data were acquired, fast Fourier transforms were calculated at $50 \%$ overlap on 256-sample Hanning windows for all artifact-free segments to transform to spectral power $\left(\mu V^{2}\right)$. Sensorimotor rhythm power was computed as the mean of $12-15 \mathrm{~Hz}$ from $\mathrm{Cz}$ and then natural log transformed (Davidson, 1988). To compute a normalized EEG power for each golfer, the relative power was used, for which the ratio of power at $12-15 \mathrm{~Hz}$ to $1-30$ Hz was computed (Niemarkt et al., 2011).

\section{Statistical Analyses}

The average putting score and standard deviation between the two groups was analyzed by a 2 (Group: SMR NFT, Control) $\times 2$ (Test: pretest, posttest) ANOVA with repeated measures on the test factor.
The difference score (posttest to pretest) for the relative power of SMR was subjected to a 2 (Group: SMR NFT, Control) $\times 3$ [Time window: -1.5 to $-1.0 \mathrm{~s}$ (T1), -1.0 to $-0.5 \mathrm{~s}$ (T2), -0.5 to $0 \mathrm{~s}$ (T3)] ANOVA with repeated measures on the time window factor.

In addition, we ran several control analyses to provide additional evidence to support our conclusions.

The success of the training ratio was tested by a 2 (Group: SMR NFT, Control) $\times 4$ (Training section: Section 1: sessions 1-2; Section 2: sessions 3-4; Section 3: sessions 5-6; Section 4: sessions 7-8) ANOVA with repeated measures on the training section.

To characterize the within-session learning effect, we compared the successful training ratio of the first and last trials of each session across all eight sessions. A 2 (Group: SMR NFT, Control) $\times 8$ (Session: session 1, 2, $3,4,5,6,7,8) \times 2$ (Trial: first trial, last trial) three-way ANOVA with repeated measures on the session, and trial was used to examine this issue.

To ensure control of neurofeedback in the SMR NFT group within the training program, we employed a one-way ANOVA with training section (Training section: Section 1: sessions 1-2; Section 2: sessions 3-4; Section 3: sessions 5-6; Section 4: sessions 7-8) as a variable to detect the threshold fluctuation within the four training sections.

To examine the regional fluctuation of $12-15 \mathrm{~Hz}$ power before and after training, we carried out a 2 (Group: SMR NFT, Control) $\times 4$ (Region: frontal, central, parietal, occipital) two-way ANOVA with repeated measures on the region.

The examination of concurrent changes in neighboring frequency bands was conducted by analyzing the pre-to-post difference scores for theta $(4-7 \mathrm{~Hz})$, alpha (8-12 Hz), low beta $(13-20 \mathrm{~Hz})$, high beta $(21-30 \mathrm{~Hz})$, and broad beta $(13-30 \mathrm{~Hz})$ frequency bands with a 2 (Group: SMR NFT, Control) $\times 3$ [Time window: -1.5 to $-1.0 \mathrm{~s}$ (T1), -1.0 to $-0.5 \mathrm{~s}(\mathrm{~T} 2),-0.5$ to $0 \mathrm{~s}$ (T3)] twoway ANOVA.

Mauchly's test was used to assess the validity of the ANOVA sphericity assumption whenever necessary. The degrees of freedom were corrected using the Greenhouse-Geisser procedure, and least significant difference analysis was used for post hoc comparisons $(p<.05)$. The partial eta square was used to estimate the effect size, with values of $.02, .12$, and .26 suggesting relatively small, medium, and large effect sizes, respectively (Cohen, 1992).

\section{Results}

\section{Putting Performance}

The mean distance of the SMR group in the pretest and posttest was $29.62 \mathrm{~cm}$ (5.59) and $16.59 \mathrm{~cm}$ (8.92), respectively. The control group distance was $20.17 \mathrm{~cm}$ (12.07) and $18.80 \mathrm{~cm}$ (5.58), respectively. An independent $t$ test showed no difference in the mean distance in the pretest between two groups $[t(14)=2.008, p=.073$, 
$\left.\eta_{p}^{2}=.224\right]$. The 2 (Group: SMR NFT, Control) $\times 2$ (Test: pretest, posttest) mixed-model ANOVA revealed a significant interaction effect on putting performance $\left[F(1,14)=5.029, p=.042, \eta^{2}=.264\right]$. The SMR neurofeedback group exhibited a shorter distance from the hole in posttest than pretest $\left[t(7)=3.417, p=.011, \eta^{2}\right.$ $=.625]$. No significant difference was observed for other comparisons.

\section{Putting Performance in Standard Deviation}

A marginal interaction effect was observed in the 2 (Group: SMR NFT, Control) $\times 2$ (Test: pretest, posttest) ANOVA $\left[F(1,14)=4.121, p=.062, \eta^{2}=.227\right]$. We did not observe an effect on Group factor $[F(1,14)=$ $\left.0.136, p=.717, \eta_{p}^{2}=.010\right]$. The SMR group exhibited a significantly lower $S D$ in the posttest $(16.11 \mathrm{~cm})$ than in the pretest $(24.70 \mathrm{~cm})\left[t(7)=4.408, p=.003, \eta_{p}^{2}=\right.$ $.735]$, whereas the control group showed no significant variation in $S D(21.03 \mathrm{~cm}$ to $18.38 \mathrm{~cm})[t(7)=1.208, p$ $\left.=.266, \eta_{p}^{2}=.173\right]$.

\section{SMR Relative Power}

The difference scores of the SMR group members for T1, $\mathrm{T} 2$, and T3 was 0.481 (0.588), 0.186 (0.378), and 0.040 (0.268), respectively. For the control group, the difference scores was $-0.200(0.424),-0.143(0.440)$, and 0.009
(0.444), respectively. We compared the difference scores with a 2 (Group: SMR NFT, Control) $\times 3$ [Time window: -1.5 to $-1.0 \mathrm{~s}$ (T1), -1.0 to $-0.5 \mathrm{~s}$ (T2), -0.5 to $0 \mathrm{~s}$ (T3)] two-way ANOVA and observed a marginally significant two-way interaction effect $[F(2,28)=3.315, p=.051$, $\left.\eta_{\mathrm{p}}^{2}=.191\right]$. To explore this marginal interaction effect and examine the training effect before and after NFT, a subsequent simple main effect analysis was performed and revealed a marginal Time effect $[F(2,14)=3.470$, $\left.p=.060, \eta_{p}^{2}=.331\right]$ in the SMR NFT group. Post hoc analysis showed that the SMR power was significantly greater in T1 than in T3 $\left[t(7)=2.925, p=.022, \eta_{p}^{2}=\right.$ .550]. No significant simple main effect was observed in the control group $\left[F(2,14)=.671, p=.567, \eta^{2}=.141\right]$. In addition, a simple main effect analysis revealed that the SMR NFT group exhibited a relatively higher SMR power than that of the control at T1 $\left[t(14)=2.657, p=.019, \eta^{2}\right.$ $=335]$. The significant group main effect revealed that the SMR NFT group had a higher SMR power than that of the control group $\left[F(1,14)=4.665, p=.049, \eta^{2}{ }_{p}=\right.$ $.250]$. The difference scores between the two groups are depicted in Figure 1.

\section{Control Analyses}

Successful Training Ratio. The overall mean of the golfers' successful training ratio was 62.39 (8.88) \% for the SMR training group and 22.27 (22.28) \% for the control group. The 2 (Group: SMR NFT, Control) $\times 4$

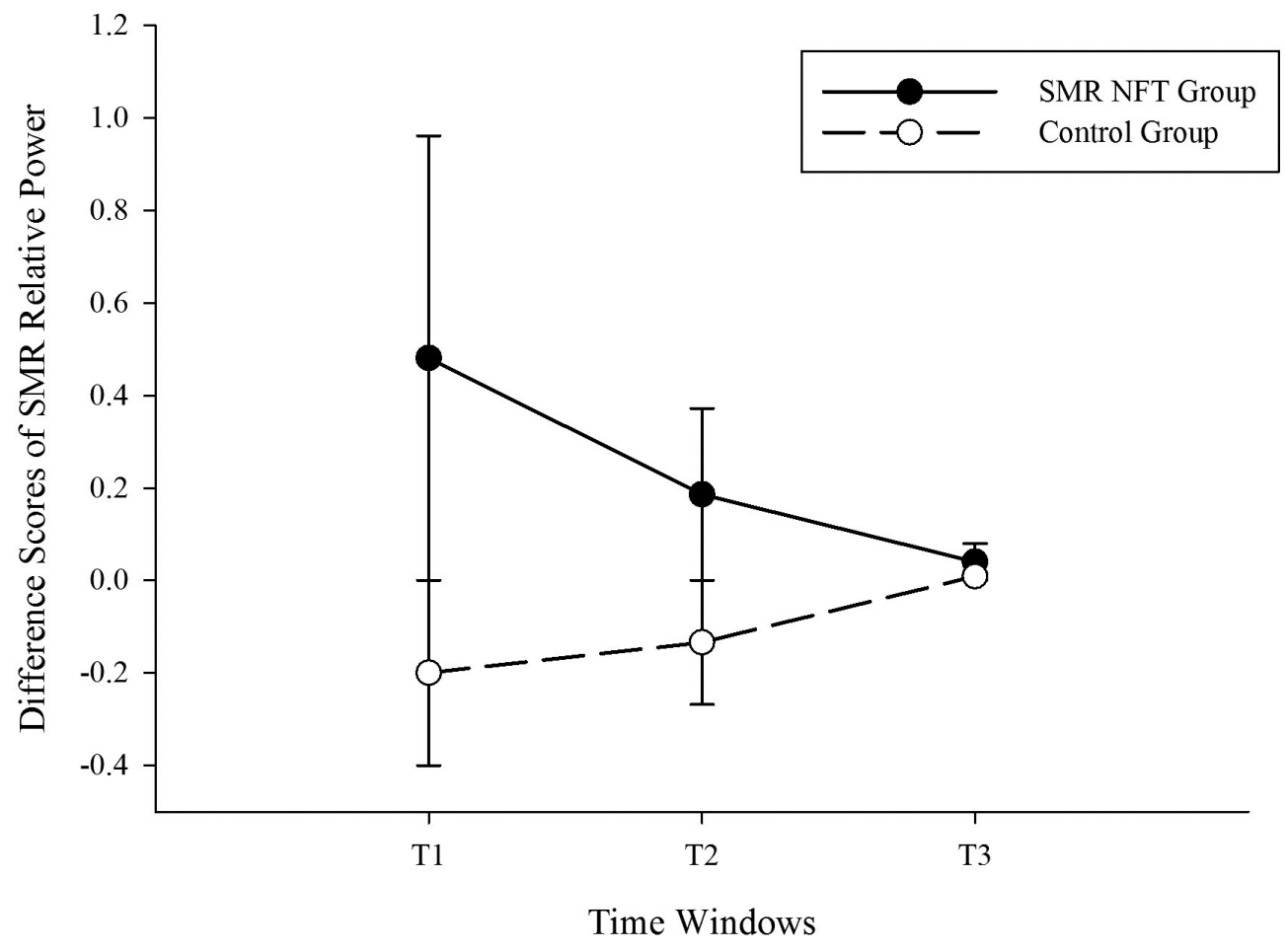

Figure 1 - The difference scores of SMR relative power between the SMR NFT and control groups at T1 ( -1.5 to $-1.0 \mathrm{~s})$, T2 ( -1.0 to $-0.5 \mathrm{~s})$, and T3 ( -0.5 to $0 \mathrm{~s})$. 
(Training section: Section 1: sessions 1-2; Section 2: sessions 3-4; Section 3: sessions 5-6; Section 4: sessions 7-8) ANOVA showed no interaction effect $[F(3,42)=$ $\left.0.694, p=.497, \eta_{\mathrm{p}}^{2}=.047\right]$, but a significant group main effect was observed $\left[F(1,14)=22.188, p=.001, \eta^{2}{ }_{p}=\right.$ .613]. The SMR group showed a significantly higher percentage of successful training ratios than did the control group. Table 1 lists the successful training ratio for each group during the training sections.

\section{Within-Session Learning.}

The results of NFT can be affected by day-to-day fluctuations in arousal level (Gruzelier et al., 2014). Thus, in addition to comparing the average successful training ratios of the eight sessions between these two groups, we compared the successful training ratios of the first and last trials of each session for all eight sessions between the two groups to determine whether participants in the NFT group improved within each training session. We hypothesized that the successful training ratio would be greater in the last trial than in the first trial for the SMR NFT group but not for control group. A 2 (Group: SMR NFT, Control) $\times 8$ (Session: sessions $1,2,3,4,5,6,7$, $8) \times 2$ (Trial: first trial, last trial) three-way ANOVA was employed to test this hypothesis. The result showed that although the 3-way interaction effect was not significant $\left[F(7,98)=2.063, p=.082, \eta^{2}=.128\right]$, a 2 (Group: SMR NFT, Control) $\times 2$ (Trial: first trial, last trial) interaction effect $\left[F(1,14)=33.192, p=.001, \eta^{2}\right.$ $=.703$ ] was revealed. Post hoc analysis was consistent with our prediction; only the SMR NFT group demonstrated a greater successful training ratio in the last trial $(M=$ 77.65, $S D=7.84)$ than in the first trial $(M=50.58, S D=$ $10.65)$ for all sessions $\left[t(7)=8.344, p=.001, \eta_{p}^{2}=909\right]$. The control group did not show a significant difference between the first trial $(M=12.19, S D=11.86)$ and last trial $(M=16.32, S D=17.00)\left[t(7)=1.784, p=.118, \eta^{2}{ }_{p}\right.$ $=313$ ]. In addition, the SMR NFT group demonstrated a significantly higher training ratio on the first trial $[t(7)=$ $\left.6.810, p=.001, \eta^{2}{ }_{p}=768\right]$ and last trial $[t(7)=9.267, p$ $\left.=.001, \eta_{\mathrm{p}}^{2}=.860\right]$ than did the control group (Figure 2).

Threshold Increments Within SMR Training Sessions. Although our control analyses provided supportive evidence for the learning progress made by the SMR NFT group, we further analyzed the change in threshold during each session of SMR NFT. In our study, threshold level was used as a difficulty index in the SMR NFT group, in which golfers were instructed to increase the SMR above designated level to meet our training demand. Thus, an improvement in the successful training ratio from the two previous control analysis was meaningful only when the threshold for each session was also examined. Previous studies evaluated the threshold variation within day-to-day sessions and suggested that the increased threshold could serve as a marker for improvement of the controllability due to neurofeedback training (Doppelmayr \& Weber, 2011). Thus, we converted the eight training sessions into four sections as described in the methods section and examined the training threshold variation by employing an one-way ANOVA to examine the effect of Training section (Section 1: sessions 1-2; Section 2: sessions 3-4; Section 3: sessions 5-6; Section 4: sessions 7-8) in the SMR group. We hypothesized that the threshold value would increase after the first training section, which supports an improvement in controllability due to SMR neurofeedback training. The average training thresholds for sections one to four in the SMR NFT group were 5.862 (2.781), 7.636 (3.368), 8.214 (3.718), and 7.750 (3.816), respectively. As predicted, a significant difference was detected by the one-way ANOVA $[F(3$, $\left.18)=9.945, p=.001, \eta_{p}^{2}=.624\right]$. Post hoc analysis demonstrated that the training thresholds in the second, third, and fourth sections were significantly higher than that of the first section.

Electrode Specificity. Although the current study demonstrated that the relative SMR power of the SMR NFT group was significantly higher than that of the control group following SMR NFT, it remained unknown whether the greater 12-15 Hz EEG relative power after training was limited to the sensorimotor cortex or there was a spillover to other regions, such as the frontal, parietal and occipital cortices. Thus, we compared the difference scores at 12-15 Hz EEG relative power among Fz, Cz, Pz, and $\mathrm{Oz}$ between pre- and posttest sessions. Previous work has shown that the SMR originated in the centro-parietal region (Grosse-Wentrup, Schölkopf, \& Hill, 2011). Thus, we hypothesized that the difference score of $12-15 \mathrm{~Hz}$ at $\mathrm{Cz}$ would be greater than that of the frontal and occipital regions for SMR group participants after training. A 2 (Group: SMR NFT, Control) $\times 4$ (Region: Frontal, Central, Parietal, Occipital) two-way ANOVA between the two groups was performed to test this hypothesis.

The difference scores at $\mathrm{Fz}, \mathrm{Cz}, \mathrm{Pz}$, and $\mathrm{Oz}$ were 0.035 (0.200), $0.212(0.178), 0.135$ (0.298), and 0.003 (0.241), respectively, for the SMR NFT group. For the

Table 1 The Successful Training Ratios Between the SMR NFT
and Control Groups Across the Four Training Sections
(Every Two Consecutive Sessions Were Folded Resulting In Four Sections)
\begin{tabular}{lccccc}
\multicolumn{7}{c}{ Section 1} & Section $\mathbf{2}$ & Section 3 & Section 4 & Total \\
\hline SMR & $53.82(19.71)$ & $63.85(12.53)$ & $65.63(9.52)$ & $66.27(17.91)$ & $62.39(5.08)$ \\
Control & $20.51(24.11)$ & $23.02(26.31)$ & $22.94(21.58)$ & $22.62(19.61)$ & $22.27(1.09)$ \\
\hline
\end{tabular}

Note. The unit is the percentage of increasing time for successfully controlling SMR power. 


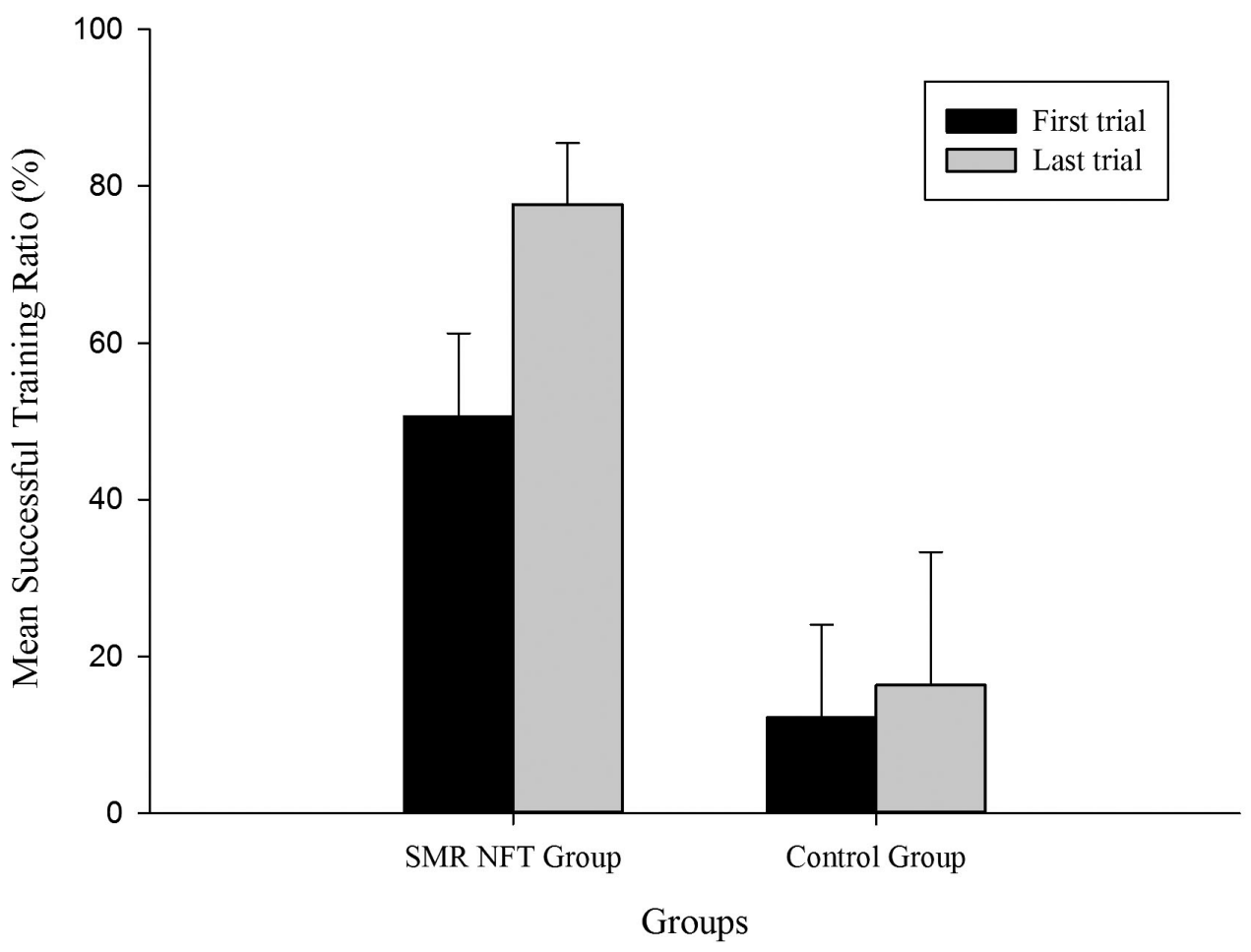

Figure 2 - The mean successful training ratio for the first and last trial between the SMR NFT and control groups across the eight training sessions.

control group, the difference scores at $\mathrm{Fz}, \mathrm{Cz}, \mathrm{Pz}$, and $\mathrm{Oz}$ were $-0.056(0.309),-0.438$ (0.169), -0.150 (0.268), and $-0.168(0.640)$, respectively. This result yielded a marginally significant interaction effect $[F(3,42)=$ $\left.2.680, p=.089, \eta^{2}=.161\right]$. Because of the exploratory nature of this study, we conducted a follow-up analysis of this interaction effect. The independent $t$ tests of the four regions between the two groups showed that significance was only observed at a difference score of $\mathrm{Cz}\left[t(14)=5.159, p=.001, \eta_{\mathrm{p}}^{2}=655\right]$, in which the SMR NFT group exhibited a significantly higher difference score than the control group. Moreover, oneway ANOVA of four regions in the SMR NFT group reached marginal significance $[F(3,21)=2.644, p=$ $\left..076, \eta_{\mathrm{p}}^{2}=.274\right]$. The follow-up pairwise $t$ tests found that the difference score of $\mathrm{Cz}$ was higher than that of $\mathrm{Fz}$ $\left[t(7)=3.740, p=.007, \eta_{p}^{2}=666\right]$ and $\mathrm{Oz}[t(7)=2.530$, $\left.p=.039, \eta_{\mathrm{p}}^{2}=.478\right]$. These lines of evidence provide preliminary support for the electrode specificity of SMR NFT in this study.

Frequency Specificity. Previous studies have shown that neurofeedback training may generate concurrent changes in flanking frequency bands (Enriquez-Geppert et al., 2014). The aim of this analysis was to investigate whether SMR NFT resulted in a change in frequency bands close to SMR. We compared the relative power difference scores of theta $(4-7 \mathrm{~Hz})$, alpha $(8-12 \mathrm{~Hz})$, low beta $(13-20 \mathrm{~Hz})$, high beta $(21-30 \mathrm{~Hz})$, and broad beta $(13-30 \mathrm{~Hz})$ frequency bands before golf putting from pretest and posttest between the two groups. The 2 (Group: SMR NFT, Control) $\times 3$ [Time window: -1.5 to $-1.0 \mathrm{~s}$ (T1), -1.0 to $-0.5 \mathrm{~s}$ (T2), -0.5 to $0 \mathrm{~s}$ (T3)] twoway ANOVA showed that neither interaction effects on theta power $\left[F(2,28)=0.550, p=.583, \eta^{2}=.038\right]$, alpha power $\left[F(2,28)=0.113, p=.802, \eta^{2}=.011\right]$, low beta power $\left[F(2,28)=0.052, p=.949, \eta^{2}=.004\right]$, high beta power $\left[F(2,28)=0.503, p=.496, \eta^{2}=.035\right]$, and broad beta band $\left[F(2,28)=0.883, p=.425, \eta_{p}^{2}=\right.$ $.059]$ nor group main effects on theta power $[F(1,14)=$ $\left.0.032, p=.860, \eta_{p}^{2}=.002\right]$, alpha power $[F(1,14)=$ $\left.0.070, p=.795, \eta_{\mathrm{p}}^{2}=.005\right]$, low beta power $[F(1,14)$ $\left.=0.764, p=.397, \eta^{2}{ }_{p}=.052\right]$, high beta power $[F(1$, $\left.14)=0.677, p=.424, \eta_{\mathrm{p}}^{2}=.046\right]$, and broad beta power $\left[F(1,14)=0.023, p=.881, \eta^{2}=.002\right]$ were observed. The difference scores among these five frequency bands are listed in Table 2.

\section{Discussion}

The aim of this study was to investigate the effect of SMR neurofeedback training on golf putting performance. Our results showed that golfers receiving SMR neurofeedback training demonstrated enhanced SMR activity during the final $1.5 \mathrm{~s}$ before golf putting, resulting in better putting performance compared with the control group. This finding lends preliminary support to the hypothesis that SMR NFT is effective for increasing SMR power, and leads to superior putting performance. 
Table 2 Difference Scores (\%) of Relative Power for Theta, Alpha, Low Beta, High Beta, and Beta Frequency Bands in Three Time Windows Between the Two Groups, SMR and Control

\begin{tabular}{|c|c|c|c|c|c|c|}
\hline \multirow[b]{2}{*}{ Relative Power } & \multicolumn{2}{|c|}{$\mathrm{T} 1(-1.5$ to $-1.0 \mathrm{~s})$} & \multicolumn{2}{|c|}{$\mathrm{T} 2(-1.0$ to $-0.5 \mathrm{~s})$} & \multicolumn{2}{|c|}{$\mathrm{T} 3(-0.5$ to $0 \mathrm{~s})$} \\
\hline & SMR & Control & SMR & Control & SMR & Control \\
\hline Theta & $.025(.621)$ & $.338(.493)$ & $-.234(.172)$ & $-.186(.528)$ & $.311(1.071)$ & $.085(.452)$ \\
\hline Alpha & $.006(.134)$ & $.048(.221)$ & $.052(.177)$ & $.017(.216)$ & $-.006(.465)$ & $-.058(.223)$ \\
\hline Low beta & $.035(.258)$ & $.014(.135)$ & $-.069(.124)$ & $-.029(.164)$ & $-.097(.183)$ & $-.033(.082)$ \\
\hline High beta & $.014(.190)$ & .015 (.109) & $-.046(.085)$ & $.128(.593)$ & $-.047(.152)$ & $-.030(.070)$ \\
\hline Beta & $.034(.164)$ & $.053(.010)$ & $-.064(.094)$ & $-.029(.077)$ & $-.050(.137)$ & $-.082(.112)$ \\
\hline
\end{tabular}

Increased SMR power by NFT results in better visuomotor performance. For behavioral data, we observed that SMR neurofeedback training improved skilled golfers' putting performance, as indicated by the reduced average distance from the hole and the variability of the score. No significant change in putting performance was observed in the control group. Previous studies have demonstrated that augmenting SMR by NFT improved visual motor performance (Ros et al., 2009) and increased self-rating scores of subjective flow state in dancers (Gruzelier et al., 2010). Furthermore, augmenting SMR by NFT was related to an improved attention-related mental state (Vernon et al., 2003) and memory performance (Hoedlmoser et al., 2008). In addition, converging lines of evidence support the effectiveness of NFT based on non-SMR variables enhancing performance in the sport domain (Arns et al., 2008; Gruzelier et al., 2010; Kao et al., 2014; Landers et al., 1991; Raymond, Sajid, Parkinson, \& Gruzelier, 2005; Ring, Cooke, Kavussanu, McIntyre, \& Masters, 2015). Nevertheless, the current study is the first, to our best knowledge, to use the SMR protocol to investigate the effectiveness of NFT on sport performance. Our results support the finding of the augmented SMR power which is linked with more adaptive fine-motor performance (Cheng et al., 2015) and extend the potential facilitation effects of SMR training to the sport domain.

Less task-irrelevant interference of somatosensory and sensorimotor processing, as reflected in augmented SMR power after training, leads to improved putting performance. A previous study has indicated that participants in the automatic stage showed weaker activity in the presupplementary motor area, premotor cortex, parietal cortex, and prefrontal cortex compared with novices in a self-paced sequential finger movement task (Wu et al., 2008). A negative relationship between SMR power and sensorimotor activity has been suggested (Mann et al., 1996). The drop in sensorimotor activity, as reflected by increased SMR power, may indicate a greater adaptive task-related attention allocation that facilitates the execution of sport performance (Gruzelier et al., 2010). Increasing SMR power through NFT is also related to more efficient and modulated visuomotor performance (Gruzelier et al., 2010; Ros et al., 2009). These results suggest that augmenting SMR power led to an improved adjustment of somatosensory and sensorimotor pathways (Kober et al., 2014), which resulted in increased task-related attention toward specific tasks (Egner \& Gruzelier, 2001). Moreover, previous studies have suggested that enhanced SMR power leads to a relatively higher flow state (Gruzelier et al., 2010) and calming mood (Gruzelier, 2014a). Based on the functional role of SMR, these findings imply that a reduction in sensorimotor activity may lessen the conscious processing involved in motor execution, which would lead to a more conceptual automatic process (Cheng et al., 2015). This interpretation is in line with converging evidence supporting a beneficial effect of augmented SMR on focusing and sustaining attention, working memory, and psychomotor skills (Egner \& Gruzelier, 2001; Ros et al., 2009). Collectively, the superior golf putting performance observed in the present SMR NFT group might be the result of reduced somatosensory information processing before the back swing, which leads to refined golf putting performance. The interpretation that a reduction in conscious interference facilitates motor operation is in line with the concept of automatic processing proposed by Fitts and Posner (1967). However, given the relatively small sample size, future research should verify the causal relationship between augmented SMR power and finemotor performance.

Reduced cortical activity in the sensorimotor area, as reflected by the higher power of $12-15 \mathrm{~Hz}$, is sensitive to superior putting performance. First, the electrode specificity of SMR NFT was demonstrated. Although electrode specificity has been suggested to be an important step in support of the NFT training effect on the corresponding EEG component at a specific brain region (Gruzelier, 2014b), this is the first study in the area of NFT and sport performance to provide such preliminary evidence for the localized training effects. The lack of difference between $\mathrm{Cz}$ and $\mathrm{Pz}$ might suggest that this region is also part of a network associated with SMR activity in motor performance. This speculation is in line with the evidence that the parietal region is involved in processing visual-spatial information during motor performance (Del Percio et al., 2011).

Second, frequency specificity was analyzed. One might argue that enhanced putting performance was caused by variation in another frequency band at the $\mathrm{Cz}$ 
site, but this explanation is inconsistent with the lack of significant changes on difference scores in the theta, alpha, low beta and high beta frequency bands. These results suggest that it is primarily SMR power that accounts for the facilitating effect of SMR NFT on putting performance rather than other neighboring frequency bands. Our demonstration of electrode and frequency specificity strengthens the hypothesis that improved putting performance was the result of reduced sensorimotor activity before putting execution.

The SMR NFT group improved the putting performance through the refined strategy for controlling the SMR power and reached the training goal as a result of the training program. First, our data showed that the SMR group demonstrated a higher successful training ratio than did the control group. Second, previous studies proposed that the training effect would emphasize daily training improvement (Gruzelier et al., 2014). In our control analysis, we compared the successful training ratio of the first and the last trial within eight sessions. A significantly higher successful training ratio for the last trial than for the first trial was observed, suggesting that golfers in the SMR NFT group learned the tuning strategy successfully after the initial trials and that the strategies were effective in the subsequent trials of the remaining sessions. This result lends support to the concept of neurofeedback trainability and further confirms the possibility of EEG tuning within a single training session (Kao et al., 2014; López-Larraz, Escolano, \& Minguez, 2012). Furthermore, we found a significant threshold increase after the first session only in SMR NFT group, suggesting that our training protocol is facilitative to golfers. This evidence was in line with previous work in which the SMR amplitude increased above the daily adjusted threshold (Weber, Köberl, Frank, \& Doppelmayr, 2011).

We have several suggestions with regard to future neurofeedback studies. First, combining these studies with neuroimaging tools is necessary. Although we have provided evidence that the regulation of SMR power can enhance putting performance, this result would be benefit from the experiments conducted with high-spatialresolution neuroimaging tools, such as fMRI, to provide a more precise anatomical description of the NFT effect. Second, the phenomenological report of neurofeedback learning and its effects is often overlooked (Gruzelier, 2014b). A sophisticated measurement of subjective mental state, such as an in-depth questionnaire or scale, is needed to further elucidate the mental state associated with NFT (Gruzelier, 2014a). Third, the retention of learning driven by NFT must be examined. Thus far, this issue has received little attention, but it is critical from a practical viewpoint to determine how long the performance enhancement due to NFT lasts. Fourth, to explore the effect of SMR NFT on anticipative motor planning is needed. Future study should investigate the link between neurophysiological and cognitive processes by using the priming tests to further understand the neurocognitive architecture of golf performance. Last but not least, the changes in network dynamics after NFT should be further examined to fill the knowledge gap of cortical interaction caused by NFT. For example, the parietal and sensorimotor cortex networks are thought to be functionally relevant during motor performance (Baumeister et al., 2013).

Our findings should be interpreted with caution due to the limitations of the study. First, the sample size was limited. Some of our statistical analyses reached only marginal significance, likely due to the small sample size. Furthermore, given the exploratory nature of the study, it is reasonable to speculate implications regarding the the marginally significant effects. Second, although the neurophysiological source of the SMR could not be precisely located due to limited spatial resolution by surface EEG, the finding of a marginally significant larger SMR difference score at the $\mathrm{Cz}$ site compared with the $\mathrm{Fz}$ and $\mathrm{Oz}$ sites as well as the finding that the largest magnitude of $12-15 \mathrm{~Hz}$ differences occurred at the $\mathrm{Cz}$ site rather than other frequency bands in the SMR group provide indirect evidence to support the impact of somatosensory activity on superior putting performance after SMR NFT. Third, putting is only one of many fundamental motor skills involved in golf performance. Our results may be difficult to generalize to other golf motor skills (e.g., the drive shot and tee shot). Future studies should, therefore, examine different skills involved in golf performance to determine the generalizability of the present findings. Fourth, the skill levels of the participants may impact the effect of NFT, and caution should be exercised when generalizing these findings to golfers at other skill levels.

In conclusion, an eight-session SMR NFT exhibited a putting performance enhancement and increased SMR power in SMR NFT group compared with control group, suggesting that SMR NFT is an effective protocol for enhancing putting performance through fine-tuning somatosensory interference, as reflected by augmented SMR.

\section{Acknowledgments}

The work of Tsung-Min Hung was supported in part by the National Science Council (Taiwan) under grant NSC 98-2410H-003 -124 -MY2.

\section{References}

Arns, M., Kleinnijenhuis, M., Fallahpour, K., \& Breteler, R. (2008). Golf Performance Enhancement and Real-Life Neurofeedback Training Using Personalized EventLocked EEG Profiles. Journal of Neurotherapy, 11(4), 11-18. doi:10.1080/10874200802149656

Babiloni, C., Del Percio, C., Iacoboni, M., Infarinato, F., Lizio, R., Marzano, N., . . Eusebi, F. (2008). Golf putt outcomes are predicted by sensorimotor cerebral EEG rhythms. The Journal of Physiology, 586(1), 131-139. PubMed doi:10.1113/jphysiol.2007.141630

Baumeister, J., Von Detten, S., Van Niekerk, S.M., Schubert, M., Ageberg, E., \& Louw, Q.A. (2013). Brain activity in predictive sensorimotor control for landings: An EEG pilot 
study. International Journal of Sports Medicine, 34(12), 1106-1111. PubMed doi:10.1055/s-0033-1341437

Chatrian, G.E., Lettich, E., \& Nelson, P.L. (1985). Ten percent electrode system for topographic studies of spontaneous and evoked EEG activity. The American Journal of EEG Technology, 25, 83-92.

Cheng, M.Y., Hung, C.L., Huang, C.J., Chang, Y.K., Lo, L.C., Shen, C., \& Hung, T.M. (2015). Expert-novice differences in SMR activity during dart throwing. Biological Psychology, 110, 212-218. PubMed doi:10.1016/j.biopsycho.2015.08.003

Chuang, L.Y., Huang, C.J., \& Hung, T.M. (2013). The differences in frontal midline theta power between successful and unsuccessful basketball free throws of elite basketball players. International Journal of Psychophysiology, 90(3), 321-328. PubMed doi:10.1016/j.ijpsycho.2013.10.002

Cohen, J. (1992). A power primer. Psychological Bulletin, 112(1), 155-159. PubMed doi:10.1037/00332909.112.1.155

Davidson, R.J. (1988). EEG measures of cerebral asymmetry: conceptual and methodological issues. The International Journal of Neuroscience, 39(1-2), 71-89. PubMed doi:10.3109/00207458808985694

Del Percio, C., Iacoboni, M., Lizio, R., Marzano, N., Infarinato, F., Vecchio, F., .. . Babiloni, C. (2011). Functional coupling of parietal alpha rhythms is enhanced in athletes before visuomotor performance: a coherence electroencephalographic study. Neuroscience, 175, 198-211. PubMed doi:10.1016/j.neuroscience.2010.11.031

Doppelmayr, M., \& Weber, E. (2011). Effects of SMR and theta/ beta neurofeedback on reaction times, spatial abilities, and creativity. Journal of Neurotherapy, 15(2), 115-129. doi: 10.1080/10874208.2011.570689

Egner, T., \& Gruzelier, J. (2001). Learned self-regulation of EEG frequency components affects attention and eventrelated brain potentials in humans. Neuroreport, 12(18), 4155-4159. PubMed doi:10.1097/00001756-20011221000058

Egner, T., Strawson, E., \& Gruzelier, J. (2002). EEG signature and phenomenology of alpha/theta neurofeedback training versus mock feedback. Applied Psychophysiology and Biofeedback, 27(4), 261-270. PubMed doi:10.1023/A:1021063416558

Egner, T., Zech, T.F., \& Gruzelier, J. (2004). The effects of neurofeedback training on the spectral topography of the electroencephalogram. Clinical Neurophysiology, 115(11), 2452-2460. PubMed doi:10.1016/j.clinph.2004.05.033

Enriquez-Geppert, S., Huster, R.J., Scharfenort, R., Mokom, Z.N., Zimmermann, J., \& Herrmann, C.S. (2014). Modulation of frontal-midline theta by neurofeedback. Biological Psychology, 95(1), 59-69. PubMed doi:10.1016/j.biopsycho.2013.02.019

Fitts, P.M., \& Posner, M.I. (1967). Human Performance. Basic Concepts in Psychology. Retrieved from http://lccn.loc. gov/67011662

Grosse-Wentrup, M., Schölkopf, B., \& Hill, J. (2011). Causal influence of gamma oscillations on the sensorimotor rhythm. NeuroImage, 56(2), 837-842. PubMed doi:10.1016/j.neuroimage.2010.04.265
Gruzelier, J. (2014a). Differential effects on mood of 12-15 (SMR) and 15-18 (beta1) Hz neurofeedback. International Journal of Psychophysiology, 93(1), 112-115. PubMed doi:10.1016/j.ijpsycho.2012.11.007

Gruzelier, J. (2014b). EEG-neurofeedback for optimising performance. III: A review of methodological and theoretical considerations. Neuroscience and Biobehavioral Reviews, 44, 159-182. PubMed doi:10.1016/j.neubiorev.2014.03.015

Gruzelier, J., Foks, M., Steffert, T., Chen, M.J.L., \& Ros, T. (2014). Beneficial outcome from EEG-neurofeedback on creative music performance, attention and well-being in school children. Biological Psychology, 95(1), 86-95. PubMed doi:10.1016/j.biopsycho.2013.04.005

Gruzelier, J., Inoue, A., Smart, R., Steed, A., \& Steffert, T. (2010). Acting performance and flow state enhanced with sensory-motor rhythm neurofeedback comparing ecologically valid immersive VR and training screen scenarios. Neuroscience Letters, 480(2), 112-116. PubMed doi:10.1016/j.neulet.2010.06.019

Hoedlmoser, K., Pecherstorfer, T., Gruber, G., Anderer, P., Doppelmayr, M., Klimesch, W., \& Schabus, M. (2008). Instrumental conditioning of human sensorimotor rhythm (12-15 Hz) and its impact on sleep as well as declarative learning. Sleep, 31(10), 1401-1408. PubMed

Kao, S.C., Huang, C.J., \& Hung, T.M. (2013). Frontal midline theta is a specific indicator of optimal attentional engagement during skilled putting performance. Journal of Sport \& Exercise Psychology, 35(5), 470-478 Retrieved from http://www.ncbi.nlm.nih.gov/pubmed/24197715. PubMed

Kao, S.C., Huang, C.J., \& Hung, T.M. (2014). Neurofeedback training reduces frontal midline theta and improves putting performance in expert golfers. Journal of Applied Sport Psychology, 26(3), 271-286. doi:10.1080/104132 00.2013 .855682

Klimesch, W., Doppelmayr, M., Pachinger, T., \& Ripper, B. (1997). Brain oscillations and human memory: EEG correlates in the upper alpha and theta band. Neuroscience Letters, 238(1-2), 9-12. PubMed doi:10.1016/S03043940(97)00771-4

Kober, S.E., Witte, M., Stangl, M., Väljamäe, A., Neuper, C., \& Wood, G. (2014). Shutting down sensorimotor interference unblocks the networks for stimulus processing: An SMR neurofeedback training study. Clinical Neurophysiology. PubMed

Krane, V., \& Williams, J.M. (2006). Psychological characteristics of peak performance. In J.M. Williams (Ed.), Applied sport psychology: Personal growth to peak performance. New York: McGraw-Hill.

Landers, D.M., Petruzzello, S.J., Salazar, W., Crews, D.J., Kubitz, K.A., Gannon, T.L., \& Han, M. (1991). The influence of electrocortical biofeedback on performance in pre-elite archers. Medicine and Science in Sports and Exercise, 23(1), 123-129. PubMed doi:10.1249/00005768199101000-00018

Logan, G.D., Hockley, W.E., \& Lewandowsky, S. (1991). Automaticity and memory. In W.E. Hockley \& S. Lewandowsky (Eds.), Relating Theory and Data: Essays on Human 
Memory in Honor of Bennet B. Murdock (pp. 347-366). East Sussex, UK: Psychology Press.

López-Larraz, E., Escolano, C., \& Minguez, J. (2012). Upper alpha neurofeedback training over the motor cortex increases SMR desynchronization in motor tasks. Proceedings of the Annual International Conference of the IEEE Engineering in Medicine and Biology Society, EMBS, 2012, 4635-4638. http://doi.org/doi:10.1109/ EMBC.2012.6347000

Mann, C.A., Sterman, M.B., \& Kaiser, D.A. (1996). Suppression of EEG rhythmic frequencies during somato-motor and visuo-motor behavior. International Journal of Psychophysiology, 23(1-2), 1-7. PubMed doi:10.1016/01678760(96)00036-0

Nicholls, A.R. (2007). A longitudinal phenomenological analysis of coping effectiveness among Scottish international adolescent golfers. European Journal of Sport Science, 7(3), 169-178. doi:10.1080/17461390701643034

Niemarkt, H.J., Jennekens, W., Pasman, J.W., Katgert, T., Van Pul, C., Gavilanes, A.W.D., . . A Andriessen, P. (2011). Maturational changes in automated EEG spectral power analysis in preterm infants. Pediatric Research, 70(5), 529-534. PubMed doi:10.1203/PDR.0b013e31822d748b

Nierhaus, T., Forschack, N., Piper, S.K., Holtze, S., Krause, T., Taskin, B., . . . Villringer, A. (2015). Imperceptible somatosensory stimulation alters sensorimotor background rhythm and connectivity. The Journal of Neuroscience, 35(15), 5917-5925. PubMed doi:10.1523/JNEUROSCI.3806-14.2015

Pelz, D., \& Frank, J.A. (2000). Dave Pelz's putting bible: The complete guide to mastering the green. New York, NY: Doubleday.

Raymond, J., Sajid, I., Parkinson, L.A., \& Gruzelier, J. (2005). Biofeedback and dance performance: A preliminary investigation. Applied Psychophysiology and Biofeedback, 30(1), 65-73. PubMed doi:10.1007/s10484-005-2175-x

Ring, C., Cooke, A., Kavussanu, M., McIntyre, D., \& Masters, R. (2015). Investigating the efficacy of neurofeedback training for expediting expertise and excellence in sport. Psychology of Sport and Exercise, 16, 118-127. doi:10.1016/j.psychsport.2014.08.005

Ros, T., Moseley, M.J., Bloom, P.A., Benjamin, L., Parkinson, L.A., \& Gruzelier, J. (2009). Optimizing microsurgical skills with EEG neurofeedback. BMC Neuroscience, 10(1), 87. PubMed doi:10.1186/1471-2202-10-87

Sauseng, P., Hoppe, J., Klimesch, W., Gerloff, C., \& Hummel, F.C. (2007). Dissociation of sustained attention from central executive functions: Local activity and interregional connectivity in the theta range. The European Journal of Neuroscience, 25(2), 587-593. PubMed doi:10.1111/ j.1460-9568.2006.05286.x

Schneider, W., \& Shiffrin, R.M. (1977). Controlled and automatic human information processing: I. Detection, search, and attention. Psychological Review, 84(1), 1-66. doi:10.1037/0033-295X.84.1.1

Semlitsch, H.V., Anderer, P., Schuster, P., \& Presslich, O. (1986). A solution for reliable and valid reduction of ocular artifacts, applied to the P300 ERP. Psychophysiology, 23(6), 695-703. PubMed doi:10.1111/j.1469-8986.1986. tb00696.x

Vernon, D., Egner, T., Cooper, N., Compton, T., Neilands, C., Sheri, A., \& Gruzelier, J. (2003). The effect of training distinct neurofeedback protocols on aspects of cognitive performance. International Journal of Psychophysiology, 47(1), 75-85. PubMed doi:10.1016/S01678760(02)00091-0

Weber, E., Köberl, A., Frank, S., \& Doppelmayr, M. (2011). Predicting successful learning of SMR neurofeedback in healthy participants: Methodological considerations. Applied Psychophysiology and Biofeedback, 36(1), 37-45. PubMed doi:10.1007/s10484-010-9142-x

Wu, T., Chan, P., \& Hallett, M. (2008). Modifications of the interactions in the motor networks when a movement becomes automatic. The Journal of Physiology, 586(Pt 17), 4295-4304. PubMed doi:10.1113/jphysiol.2008.153445

Manuscript submitted: June 30, 2015

Revision accepted: October 08, 2015 